daß die Grenzen dieser Cyclobutenon-Synthese die der KetenCycloaddition sind.

Tabelle 1. Cyclobutenone (2)-(9) aus 3-Äthoxy-cyclobutanonen vom Typ (1) mit Aluminiumoxid.

\begin{tabular}{lllll}
\hline Verb. & $\mathrm{R}^{1}$ & $\mathrm{R}^{2}$ & $\mathrm{R}^{3}$ & Ausb. [\%] \\
\hline (2) & $\mathrm{C}_{6} \mathrm{H}_{3}$ & $\mathrm{C}_{6} \mathrm{H}_{3}$ & $\mathrm{H}$ & $15-35$ \\
$(3)$ & $\mathrm{C}_{6} \mathrm{H}_{5}$ & $\mathrm{C}_{6} \mathrm{H}_{3}$ & $\mathrm{CH}_{3}$ & $65-80$ \\
$(4)$ & $\mathrm{C}_{6} \mathrm{H}_{5}$ & $\mathrm{C}_{6} \mathrm{H}_{5}$ & $\mathrm{C}_{2} \mathrm{H}_{5}$ & 86 \\
$(5)$ & $\mathrm{C}_{6} \mathrm{H}_{5}$ & $\mathrm{C}_{6} \mathrm{H}_{3}$ & $\left.\mathrm{CH}_{3} \mathrm{CH}_{3}\right)_{2}$ & 58 \\
$(6)$ & $\mathrm{C}_{6} \mathrm{H}_{5}$ & $\mathrm{CH}_{3}$ & $\mathrm{CH}_{3}$ & 56 \\
$(7)$ & $\mathrm{C}_{6} \mathrm{H}_{3}$ & $\mathrm{C}_{2} \mathrm{H}_{5}$ & $\mathrm{CH}_{3}$ & 55 \\
$(8)$ & $\mathrm{C}_{6} \mathrm{H}_{5}$ & $\mathrm{CH}\left(\mathrm{CH}_{3}\right)_{2}$ & $\mathrm{CH}_{3}$ & 70 \\
(9) & $\mathrm{CH}_{3}$ & $\mathrm{CH}_{3}$ & $\mathrm{CH}_{3}$ & 75 \\
\hline
\end{tabular}

Die Cyclobutenone geben sich durch die $\mathrm{C}=\mathrm{O}$-Schwingung bei 1741-1758 $\mathrm{cm}^{-1}$, durch die UV-Absorption $\left(\mathrm{n} \rightarrow \pi^{*} \approx\right.$ $320 \mathrm{~nm}, \pi \rightarrow \pi^{*} \leqslant 227 \mathrm{~nm}$ ) sowie ${ }^{1} \mathrm{H}-\mathrm{NMR}$-Spektren $\left(\tau_{3-H}=\right.$ 1.42-2.07) zu erkennen. (2) wurde zum bekannten 2,2-Diphenyl-cyclobutanon ${ }^{[4]}$ katalytisch hydriert. Während (6)(9) im Vakuum destilliert werden konnten, zersetzten sich die kristallinen Cyclobutenone (2)-(5) schon bei Raumtemperatur.

\title{
2-Äthyl-4,4-diphenyl-2-cyclobutenon (4)
}

$1.10 \mathrm{~g}$ (3.74 mmol) 3-Äthoxy-4-äthyl-2,2-diphenyl-cyclobutanon in $10 \mathrm{ml}$ wasserfreiem Äther trug man auf eine Säule $\left(2 \mathrm{~cm}\right.$ Durchmesser) mit $70 \mathrm{~g} \mathrm{Al}_{2} \mathrm{O}_{3}$ (Woelm, Akt.-Stufe I) auf, eluierte mit $280 \mathrm{ml}$ Äther und entfernte das Solvens am Rotationsverdampfer; Ausbeute $0.80 \mathrm{~g}(85 \%)$ kristallines (4).

\section{2,4,4-Trimethyl-2-cyclobutenon (9)}

$19.0 \mathrm{~g}(0.12 \mathrm{~mol}) 3-\ddot{A}$ thoxy-2,2,4-trimethyl-cyclobutanon in $290 \mathrm{ml}$ Äther wurden mit $230 \mathrm{~g}$ neutralem $\mathrm{Al}_{2} \mathrm{O}_{3}$ angeteigt; nach $24 \mathrm{~h}$ goß man den Brei auf eine Säule mit $100 \mathrm{~g}$ trockenem $\mathrm{Al}_{2} \mathrm{O}_{3}$ und eluierte mit $800 \mathrm{ml}$ Äther. Das Solvens wurde über eine Kolonne abdestilliert; (9) ging bei $49-51^{\circ} \mathrm{C} / 28$ Torr als farblose, stechend riechende Flüssigkeit über; Ausbeute $10.0 \mathrm{~g}(75 \%)$.

Eingegangen am 16. April 1975 [Z 233a]

\section{Von Herbert Mayr und Rolf Huisgen ${ }^{[*]}$}

3-Alkoxy-cyclobutanone sind durch [2+2]-Cycloaddition von Ketenen an Enoläther bequem zugänglich ${ }^{[1]}$. Bei der Säulenchromatographie an neutralem oder basischem Aluminiumoxid der Aktivitätsstufe I spalten die Addukte (1) Äthanol ab und liefern die reinen Cyclobutenone (2)-(9) (Tabelle 1). Die aktiven Stellen des Kontakts - es wird eine polyfunktionelle Katalyse vermutet - werden durch den Alkohol blockiert. Um vollständigen Umsatz zu erreichen, benötigt man pro mmol (1) ca. 10-30 g Aluminiumoxid.

Wenn man von der Additionsrichtung absieht, sind (2)-(9) formale Produkte der Keten-Addition an Alkine; letztere Reaktion ist nur mit elektronen-anziehend substituierten Ketenen durchführbar ${ }^{[2]}$. Auf dem Umweg über (1) nutzt man die den Alkinen weit überlegene ketenophile Aktivität der

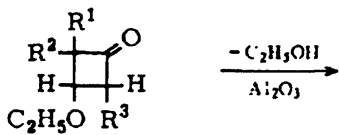

(1)

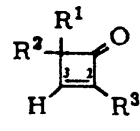

(2) $-(9)$

Enoläther ${ }^{[3]}$. Da die Alkohol-Eliminierung bei allen bisher geprïften Keten-Enoläther-Addukten gelang, nehmen wir an,

[*] Dr. H. Mayr und Prof. R. Huisgen [*]

Institut für Organische Chemie der Universität

8 München 2. Karlstraße 23

$\left[{ }^{+}\right]$Korrespondenzautor. 